\title{
Percepções de gestores municipais sobre ações de promoção da saúde: em foco os agentes comunitários de saúde'
}

\section{Municipal managers' perceptions on health promotion actions: community health workers in focus}

\author{
Lislaine Aparecida Fracolli \\ Doutora em Enfermagem. Docente do Departamento de Enferma- \\ gem em Saúde Coletiva da Escola de Enfermagem da Universidade \\ de São Paulo. \\ Endereço: Av. Dr. Enéas de Carvalho Aguiar, 419, CEP 05403-000, \\ São Paulo, SP, Brasil. \\ E-mail: lislaineœusp.br \\ Maria Fernanda Pereira Gomes \\ Doutoranda em Ciências da Saúde na Escola de Enfermagem da \\ Universidade de São Paulo. Docente da Universidade Paulista. \\ Endereço: Rua Myrtes Spera Conceição, 30I, CEP 19813-550, Assis, \\ SP, Brasil. \\ E-mail: mferpg®usp.br \\ Anna Luiza de Fátima Pinho Lins Gryschek \\ Doutora em Enfermagem. Docente do Departamento de Enferma- \\ gem em Saúde Coletiva da Escola de Enfermagem da Universidade \\ de São Paulo. \\ Endereço: Av. Dr. Enéas de Carvalho Aguiar, 419, CEP 05403-000, \\ São Paulo, SP, Brasil. \\ E-mail: gryschekळusp.br \\ I Pesquisa financiada pela Fundação de Amparo à Pesquisa do \\ Estado de São Paulo (FAPESP).
}

\section{Resumo}

Considerando a importância de investigar a competência de promoção da saúde na prática dos agentes comunitários de saúde e o fato de que a visão dos gestores de saúde influencia no desenvolvimento dessa competência, este artigo descreve as percepções dos gestores de saúde sobre as ações de promoção da saúde praticadas pelos agentes comunitários de saúde. Trata-se de pesquisa qualitativa cujo cenário de estudo foi o Colegiado de Gestão Regional do Alto Capivari do Departamento Regional de Saúde de Presidente Prudente (SP). Foram sujeitos da pesquisa os secretários municipais de saúde. Os dados foram coletados por meio de um questionário com perguntas abertas em julho e agosto de 2011. As respostas foram submetidas à técnica de análise de conteúdo proposta por Bardin. Os resultados apontaram que os gestores de saúde confundem a competência promoção da saúde e prevenção com monitoramento de grupos específicos e morbidades. Também se observou que os gestores não conseguem diferenciar a Política Nacional de Promoção da Saúde e a Política Nacional de Atenção Básica. Cidades onde os gestores municipais compreendiam e diferenciavam melhor essas políticas apresentavam agentes comunitários de saúde mais engajados na realização de ações de promoção da saúde, inclusive com práticas de intersetorialidade bem estruturadas. Conclui-se que os gestores municipais de saúde desempenham um papel fundamental para a efetivação da competência de promoção da saúde pelo agente comunitário de saúde e pela equipe de Saúde da Família.

Palavras-chave: Promoção da Saúde; Gestor de Saúde; Agentes Comunitários de Saúde. 


\section{Abstract}

Considering the importance of investigating the competence of health promotion in the practice of community health workers and the fact that health managers' view influences on the development of this competence, this article describes health managers' perceptions on health promotion actions taken by community health workers. This is a qualitative research whose study setting was the Regional Management Board of Alto Capivari of the Regional Health Department of Presidente Prudente, São Paulo, Brazil. Municipal health secretaries were the research subjects. Data were collected by means of a questionnaire with open questions in July and August 2011. The answers underwent the content analysis technique proposed by Bardin. The results pointed out that health managers confuse the competence health promotion and prevention with monitoring of specific groups and morbidities. It was also observed that managers are not able to differentiate the National Policy of Health Promotion from the National Policy of Primary Care. Towns where municipal managers better understood and differentiated these policies had community health workers more committed to take health promotion actions, including well-structured inter-sector practices. We conclude that municipal health managers play a crucial role for effecting the competence of health promotion by the community health worker and the Family Health team.

Keywords: Health Promotion; Health Manager; Community Health Workers.

\section{Introdução}

A Promoção da Saúde é uma estratégia de articulação transversal, na qual se confere visibilidade aos fatores que colocam a saúde da população em risco e às diferenças entre necessidades, territórios e culturas presentes no País. A finalidade da Promoção da Saúde é a criação de mecanismos que reduzam as situações de vulnerabilidade, defendam radicalmente a equidade e incorporem a participação e o controle social na gestão das políticas públicas (Brasil, 2007).

A potencialidade do agente comunitário de saúde (ACS) para a Promoção da Saúde na Atenção Básica é dada pela sua possibilidade de capacitar a população para o enfrentamento dos problemas de saúde (Chiesa e Fracolli, 2004). Diante dessa potencialidade do ACS, cabe aos pesquisadores, cada vez mais buscarem referências interpretativas para compreender e desenvolver as ações de Promoção da Saúde na prática desse profissional.

Retomando as competências propostas pelo Brasil (2004), para o ACS, e enfocando mais especificamente as ações que compõem a competência Promoção da Saúde, é importante buscar os nexos entre as particularidades culturais e o contexto social que instauram as práticas de Promoção da Saúde realizadas pelos ACSs nos diferentes territórios. Segundo o Ministério da Saúde (Brasil, 2004) o ACS deve desenvolver cinco competências básicas, quais sejam:1) Integração da equipe com a população local; 2) Planejamento e Avaliação; 3) Promoção da Saúde; 4) Prevenção e Monitoramento de risco ambiental e sanitário e 5) Prevenção e Monitoramento a grupos específicos e morbidades.

Pesquisas desenvolvidas por Santos (2005), Almeida (2008), Pinto (2010) e Gomes (2011), sobre a frequência de realização das cinco competências propostas pelo Ministério da Saúde para o ACS, descreveram que as competências Integração da equipe de saúde com a população local e Prevenção e monitoramento a grupos específicos e morbidades foram as mais realizadas, nas pesquisas dos autores citados acima; observou-se também que a competência Promoção da Saúde é a menos realizada.

Ainda na pesquisa de Santos (2005), conduzida no município de São Bernardo do Campo, as ações de Promoção da Saúde que foram mais realizadas pelos ACSs foram: estimula os indivíduos e a comunida- 
de a refletirem sobre as suas condições de saúde e doença; orienta indivíduos quanto ao autocuidado; orienta a população quanto a medidas de proteção à saúde (alimentação, higiene pessoal, limpeza, acondicionamento e destino do lixo, cuidados com a água e dejetos e outras). No município de Marília, segundo Pinto (2010), as ações de Promoção da Saúde mais realizadas pelos ACSs são as mesmas encontradas por Gomes (2011), em sua pesquisa no Colegiado de Gestão Regional (CGR) do Alto Capivari.

Observou-se nas pesquisas realizadas por Santos (2005), Pinto (2010) e Gomes (2011), que as ações de Promoção da Saúde mais realizadas pelos ACSs são as mesmas, o que nos mostra que a orientação às famílias e comunidades sobre as questões de higiene e saúde permeiam fortemente as práticas do ACS, em relação à Promoção da Saúde.

A pesquisa realizada por Gomes (2011), no CGR do Alto Capivari, encontrou ainda que os municípios de João Ramalho e Rancharia são os que mais realizam ações de Promoção da Saúde, quando comparados com os outros municípios que compõem esse CGR.

A análise dos dados das pesquisas realizadas por Santos (2005), Pinto (2010) e Gomes (2011) mostrou que, embora os municípios onde a prática dos ACSs foi analisada sejam municípios muito diferentes entre si (município de grande porte, médio porte e pequeno porte), as competências, de modo geral, e as ações de Promoção da Saúde, de um modo específico, possuem um padrão de ocorrência muito parecido. Tal fato suscitou a busca por respostas que possam ajudar na reflexão do papel do gestor no fomento das ações de promoção da saúde. Será que as percepções dos gestores sobre a promoção da saúde e o valor e estímulo dado a essas ações pelos gestores locais de saúde pode ser um fator decisivo na direcionalidade imprimida ao trabalho do ACS?

Diante da importância de investigar a realização das ações de saúde que contemplam a competência promoção da saúde pelos ACSs, e levando-se em consideração a premissa de que a visão política dos gestores de saúde influencia no desenvolvimento das ações de promoção da saúde, o objetivo deste artigo é descrever as percepções dos gestores de saúde sobre as ações de promoção da saúde desempenhadas pelos ACSs.

\section{Metodologia}

Trata-se de uma pesquisa qualitativa, do tipo estudo de caso. Teve como cenário de estudo o CGR de Alto Capivari, pertencente ao Departamento Regional de Saúde (DRS) 11, Presidente Prudente (São Paulo, 2012). Esse CGR é composto pelos municípios de Iepê, João Ramalho, Nantes, Quatá e Rancharia. Esses municípios possuem pequeno porte e a população deles varia de 28.809 mil habitantes (Rancharia), o mais populoso, a 2.750 mil habitantes (Nantes), o menos populoso. No CGR do Alto Capivari, os municípios de Iepê, João Ramalho, Quatá e Rancharia foram classificados, em 2008, com Índice Paulista de Responsabilidade Social (IPRS) 4 e o município de Nantes, com IPRS 3. Isso mostra que a maioria dos municípios que compõem o CGR Alto Capivari estão classificados no grupo 4 do Indicador IPRS, o que indica que esses municípios apresentam níveis baixos de riqueza e níveis intermediários de longevidade e/ou escolaridade (Seade, 2012).

Foram sujeitos da pesquisa cinco secretários municipais de saúde, dos cinco municípios que compõem o CGR do Alto Capivari. Os dados foram coletados através de um questionário de perguntas abertas, que buscava identificar as percepções dos gestores sobre a estrutura de saúde dos municípios, as ações de Promoção da Saúde desenvolvidas, bem como, as potencialidades do ACS e do município para o desenvolvimento das ações de Promoção da Saúde.

O procedimento adotado para a coleta de dados foi contato telefônico inicial com os gestores, para convidá-los a participar da pesquisa. No contato foram explicados os objetivos da pesquisa e sua contribuição para os estudos no campo da Promoção da Saúde. Informava-se também que eles receberiam via e-mail, um questionário de perguntas abertas, que eles deveriam preencher e enviar para o pesquisador e o Termo de Consentimento Livre e Esclarecido (este último foi recolhido na reunião do CGR). Dos gestores municipais de saúde do CGR Alto Capivari, cinco concordaram em participar da pesquisa, sendo um de cada município do CGR. O período de coleta dos dados compreendeu o período de julho a agosto de 2011.

A técnica de análise escolhida foi a "análise de conteúdo” proposta por Bardin (1970). A análise de 
conteúdo é um conjunto de técnicas de análise das comunicações visando obter por procedimentos sistemáticos e objetivos de descrição do conteúdo das mensagens, indicadores que permitam a inferência de conhecimentos relativos às condições de produção/recepção (variáveis inferidas) destas mensagens (Bardin, 1970). A presente pesquisa foi aprovada pelo Comitê de Ética em Pesquisa da Escola de Enfermagem da Universidade de São Paulo e pelos Secretários Municipais de Saúde dos cinco municípios. O número de protocolo do parecer é 963/2010/CEP-EEUSP.

\section{Resultados e Discussão}

Para fins de análise identificaram-se os gestores de saúde dos municípios que compõem o CGR do Alto Capivari por $\mathrm{G}_{1}, \mathrm{G}_{2}, \mathrm{G}_{3}, \mathrm{G}_{4}$ e $\mathrm{G}_{5}$. Ao analisar as respostas dos gestores de saúde, as ideias foram organizadas em duas categorias de análise: A) As ações de Promoção da Saúde e sua articulação com a Política Nacional de Promoção da Saúde (PNPS); B) As ações de Promoção da Saúde que o ACS deve realizar.

\section{As ações de promoção da saúde e sua articulação com a política nacional de promoção da saúde}

No desenvolvimento desta pesquisa, visando conhecer as percepções dos gestores municipais de saúde, perguntou-se como são desenvolvidas as ações de Promoção da Saúde nesses municípios e se as ações de Promoção da Saúde que são desenvolvidas estão articuladas à PNPS.

As respostas dos gestores de saúde mostraram que eles gerenciam as ações de saúde com concepções restritas de Promoção da Saúde.

G2 - Os gestores de Saúde do município vêm aos poucos tentando construir um modelo de atenção à saúde que priorize ações de Promoção à Saúde, visando à qualidade de vida da população. $O$ modelo atual ainda espelha um assistencialismo muito grande, vinculado aos cofres públicos, e esta realidade dificulta várias ações que constituem compromissos sanitários.

G3 - Sim, elas estão articuladas à política Nacional. Algumas das ações são: Esclarecer e orientar em saúde de acordo com o que for observado nos lares;
Captação de gestantes para o pré-natal; Captação de mulheres para o preventivo; Captação de mulheres para mamografias; Captação de homens com idade preconizada pelo ministério para o exame de PSA; Captação de crianças para puericultura; Captação de hipertensos e diabéticos; Verificação da carteira de vacina.

O gestor G2 afirma que as ações de saúde desenvolvidas em seu município ainda estão pautadas no modelo médico, assistencialista e curativista, mas que os gestores de saúde de uma forma geral têm tentado reorientar as práticas para a promoção da saúde. 0 gestor $\mathrm{G}_{3}$ considera que as ações de prevenção de doenças e proteção da saúde como a realização do exame colpocitológico pelas mulheres, vacinação e ações preventivas de complicações das doenças crônico-degenerativas são ações de promoção da saúde. Observa-se que muitos gestores de saúde, profissionais de saúde e a própria população confundem as ações de promoção da saúde com as ações de prevenção de doenças, ações de controle das doenças crônico-degenerativas e ações de proteção à saúde.

A percepção dos gestores do CGR do Alto Capivari sobre as ações de Promoção da Saúde está de acordo com o conceito da história natural da doença, cuja ênfase se concentra na prevenção das doenças, procurando se antecipar e evitar que elas ocorram, a partir de ações que visam (re)estabelecer o equilíbrio dinâmico entre o hospedeiro, o agente patogênico e o meio (Czeresnia, 2003).

De acordo com uma pesquisa que foi realizada no Nordeste Brasileiro sobre municípios saudáveis, verificou-se que projetos de Promoção da Saúde são bem-sucedidos, quando incorporam práticas intersetoriais para responder às necessidades de saúde da população, superando assim as práticas desfragmentadas e voltadas para a cura das doenças sem considerar o contexto sociopolítico e cultural dos indivíduos. A intersetorialidade é uma nova forma de governar, construir e fortalecer políticas públicas capazes de superar a fragmentação dos setores, dos saberes e das estruturas governamentais e sociais, de forma a promover efeitos mais significativos na vida da sociedade civil (Melo Filho e col., 2007).

De acordo com os autores Fernandez e Mendes (2007), os projetos de Promoção da Saúde podem ter sucesso, como no caso do Projeto de "Municípios 
Saudáveis”, mas para que isso ocorra, é necessária a apropriação de grande potencial de transformação social e política pelos gestores locais. $\mathrm{O}$ gestor municipal deve exercer o papel de articulador, mobilizador e catalisador para que os efeitos das políticas públicas sejam potencializados.

Apesar de as pesquisas desenvolvidas por Sá e Nishida (2008) e Melo Filho e colaboradores (2007) demonstrarem que a intersetorialidade é o que torna os projetos de Promoção da Saúde exitosos, confronta-se essa perspectiva com a arquitetura organizacional dos equipamentos sociais e as políticas públicas que não privilegiam o desenvolvimento de ações intersetoriais.

Os gestores de saúde do CGR Alto Capivari não consideram as ações de Promoção da Saúde articuladas à Política Nacional de Promoção da Saúde. Muitos inclusive desconhecem os objetivos, diretrizes, estratégias de implementação e responsabilidades das esferas de gestão e as ações específicas que a PNPS apresenta.

A PNPS propõe como ações específicas: a alimentação saudável; prática corporal e atividade física; prevenção e controle do tabagismo; redução da morbimortalidade em decorrência do uso abusivo de álcool e outras drogas; redução da morbimortalidade por acidentes de trânsito; prevenção da violência e estímulo à cultura da paz; e a promoção do desenvolvimento sustentável. Ao se comparar a percepção dos gestores com as proposições da PNPS, é possível verificar que existe um descompasso entre a percepção dos gestores e a noção de promoção da saúde colocada na PNPS. Esta última possui uma concepção de promoção que amplia a noção reducionista de prevenção de doenças.

Com relação à diferenciação que os gestores fazem da PNPS e da Política Nacional de Atenção Básica, as respostas de dois gestores mostram desconhecimento sobre a diferença entre elas, chegando mesmo a achar que são sinônimos, ou "mais do mesmo".

G2 - A Equipe de Saúde da Família (ESF) I está um passo à frente nas ações de Promoção à Saúde, pois já desenvolve trabalhos educativos voltados à população. A ESF II ainda está centrada na figura do médico e cria uma rotina infindável de consultas, exames e medicalização.
A fragilidade demonstrada pelas respostas dos gestores de saúde do CGR do Alto Capivari, em desconhecer as Políticas de Saúde que norteiam as práticas da Atenção Básica e de Promoção da Saúde, poderia ser objeto de intervenção político-pedagógica da DRS 11 Presidente Prudente, ou do Conselho de Secretários Municipais de Saúde (COSEMS). É sabido que espaços de gestão pública muitas vezes são espaços políticos e não técnicos, mas se não se tentar aproximar essas duas dimensões da gestão pública será difícil conseguir resultados favoráveis no setor público de saúde (Ibanez e col., 2011).

\section{As ações de promoção da saúde que o ACS deve realizar}

Buscou-se conhecer a opinião dos gestores municipais sobre o fato de o ACS realizar ações de Promoção da Saúde, tentando identificar quais seriam essas ações na perspectiva dos gestores e encontrou-se que, para os gestores de saúde, as ações de Promoção da Saúde devem ser realizadas pela equipe de saúde e não somente pelos ACSs. De acordo com a PNPS de 2006, as ações de Promoção da Saúde devem ser planejadas, organizadas e operacionalizadas pelos profissionais de saúde; gestores de saúde municipais, estaduais e federais priorizando o desenvolvimento do controle e mobilização social das coletividades (Brasil, 2007).

G2 - O ACS é o grande elo de ligação entre a equipe de saúde e a população e cabe a este profissional conhecer a realidade de vida da população, e junto a outros profissionais da Equipe articular ações intersetoriais, que busquem a qualificação das ações de Promoção à Saúde.

G3 - A promoção da saúde, não é somente da responsabilidade do Agente Comunitário de Saúde, mas com certeza é a porta de entrada da saúde nos lares dos usuários.

Embora a PNPS aponte que a responsabilidade pelas ações de Promoção da Saúde também é dos gestores de saúde, essa afirmação não foi evidenciada em nenhuma das respostas dos gestores municipais. No Plano Estadual de Saúde (2012-2015), a Promoção da Saúde, que era um dos eixos prioritários no Plano Estadual anterior (2008-2011), deixa de ser um eixo prioritário, indicando um retrocesso na priorização 
da Promoção da Saúde (SES, 2012).

Outro fato que surge nos discursos dos gestores é a percepção de que as características sociais do ACS facilitam o desenvolvimento das ações de Promoção da Saúde. Essa percepção encontra consonância com as diretrizes do Ministério da Saúde, que considera que o ACS tem um papel importante no desenvolvimento dessas ações, pois suas características socioculturais facilitam a comunicação com a população e seu trabalho permite que a equipe de saúde possa conhecer os pontos de vulnerabilidades e as necessidades de saúde que as famílias apresentam (Brasil, 2007).

Tomando por base o objetivo geral da PNPS, que é de promover a qualidade de vida e reduzir a vulnerabilidade e riscos à saúde relacionados aos seus determinantes e condicionantes: modos de viver, condições de trabalho, habitação, ambiente, educação, lazer, cultura, acesso a bens e serviços essenciais, verifica-se o quanto é importante o desenho que os ACSs fazem das famílias com as quais trabalham e a potencialidade do trabalho dos ACSs para o desenvolvimento da Promoção da Saúde (Brasil, 2007).

Observa-se que a visão dos gestores sobre as ações de Promoção da Saúde é que estas devem se fundamentar na mudança de hábitos dos indivíduos e não no empoderamento da população. Essa percepção se contrapõe às proposições da PNPS, que diz que é preciso superar a abordagem individualizante e fragmentária dos modos de viver e trabalhar das populações. Esta forma de abordagem coloca os sujeitos e as comunidades como os responsáveis únicos pelas várias mudanças/arranjos ocorridos no processo saúde-adoecimento ao longo da vida (Brasil, 2007).

G1 - Já que nas visitas o mesmo orienta quanto à prevenção e cuidados com hipertensão arterial $e$ Diabetes Melito, sabendo que o intuito da Estratégia Saúde da Família é a prevenção, adotar medidas de mudanças de hábitos saudáveis como dieta, atividade física e desmedicalização.

$\mathbf{G}_{3}$ - Se atender as mudanças de comportamento dos usuários, mudando suas práticas e discursos, de modo que possam estabelecer um processo de sensibilização desses usuários.
G5 - Orientação, acompanhamento, educação popular em saúde, a partir da concepção de saúde como promoção de qualidade de vida (mudanças de hábitos).

Essa visão de estilos de vida e mudanças comportamentais individuais perpassou também a concepção dos gestores envolvidos em projetos de municípios saudáveis (Sá e Nishida, 2008).

A Política Nacional de Promoção da Saúde propõe que as intervenções de saúde ampliem seu escopo, tomando como objeto os problemas e as necessidades de saúde e seus determinantes e condicionantes, de modo que a organização da atenção e do cuidado envolva, ao mesmo tempo, as ações e os serviços que operem sobre os efeitos do adoecer e aqueles que visem ao espaço para além dos muros da unidade de saúde e do sistema de saúde, incidindo sobre as condições de vida e favorecendo a ampliação de escolhas saudáveis por parte dos sujeitos e das coletividades no território onde vivem e trabalham (Brasil, 2007).

A discussão sobre quem deve organizar as ações de promoção da saúde não obteve consenso entre os gestores. Alguns diziam que estas deveriam ser organizadas pelos ACSs, outros pela equipe de saúde e de outros equipamentos sociais, um gestor considera que as ações de Promoção da Saúde devem partir da comunidade e outros não conseguiram ter uma opinião sobre o assunto.

G1 - Trabalhamos de forma interdisciplinar, portanto os ideais partem de toda equipe de saúde.

G2 - As ações de Promoção à Saúde desenvolvidas no âmbito municipal são realizadas pela união de setores, a Saúde e a Assistência Social realizam trabalhos conjuntamente e a comunidade interage positivamente às ações desenvolvidas.

A pesquisa de Gomes (2011) mostrou que os ACSs atuam na perspectiva da quantidade e não da equidade, realizam as visitas para cumprir a meta mensal e não estão preocupados com o desenvolvimento das ações de Promoção da Saúde, que visem o empoderamento das famílias. Verificou-se também que os ACSs realizam de forma efetiva ações de vigilância, controle e prevenção de doenças, o que reflete na visão dos gestores de saúde, sobre o que deve ser a prática do ACS. 
A percepção do gestor de saúde é a visão que permeia as práticas de saúde locais, para tanto, é necessário, que os gestores sejam capacitados na perspectiva da Promoção da Saúde e preparados para operacionalizar a PNPS. A pesquisa de Gomes (2011) discute que o CGR pode ser um espaço de discussão e capacitação técnico-política dos gestores locais.

Contudo, segundo os gestores, as ações de saúde desenvolvidas pelos ACSs quase não são foco de discussão nas reuniões do CGR, pelos gestores municipais e estaduais.

G1 - Não como pauta fixa, mas em momentos oportunos, pois o que discutimos nos CGRs é a capacitação e qualificação da Estratégia Saúde da Família, mas este tema é discutido nas reuniões de equipe da saúde família que ocorrem semanalmente.

G2 - O Colegiado de Gestão Regional do Alto Capivari é um palco de diversas discussões, entre elas sobre o trabalho dos ACSs. Vários projetos estão em andamento visando à qualificação dos profissionais inseridos na Atenção Básica (AB). Em andamento estão sendo desenvolvidos os cursos Introdutório em $A B$ e o curso de Humanização, ambos voltados à ESF. Aguardamos a liberação do recurso financeiro para realização do treinamento específico para o ACS que já foi aprovado e logo estará se iniciando.

Alguns consideram que as ações desenvolvidas pelos ACSs são discutidas nas reuniões do CGR do Alto Capivari, outros acham que são pouco discutidas. Discutir sobre Educação Permanente nas reuniões mensais do CGR do Alto Capivari é um ponto positivo, já que na pesquisa desenvolvida por Gomes (2011), identificou-se que nesse CGR o percentual de ACSs capacitados para o trabalho é de 4,93\%, demonstrando no CGR em pauta que está faltando capacitação para os ACSs. O percentual baixo de capacitação dos ACSs demonstra que os gestores de saúde estão atuando de forma descompromissada com a Educação Permanente.

No desenvolvimento do trabalho do ACS, observa-se que o contato que esse profissional tem com as famílias é maior que o da equipe de saúde, já que, ao realizar a visita domiciliar mensal, tem contato com pessoas que não foram à unidade de saúde naquele mês e também com pessoas que não utilizam a unidade de saúde. Essa constatação demonstra a importância em se investir e discutir sobre as ações de saúde desenvolvidas pelos ACSs nas reuniões mensais do CGR, pois eles têm potencial para desenvolver ações com ampla cobertura da população (Santos, 2005; Gomes, 2011).

As reuniões mensais do CGR do Alto Capivari são espaços onde se discutem necessidades de capacitação e as propostas de Educação Permanente, assim é importante discutir as ações que estão sendo desenvolvidas pelos ACSs, para identificar as estratégias de formação necessárias para esses profissionais desenvolverem sua tarefa. Os gestores de saúde devem saber claramente o que esperam das ações de saúde desenvolvidas pelos ACSs e o que querem impactar na saúde da população, com o desenvolvimento das ações do profissional ACS.

De acordo com a PNPS, é urgente superar a cultura administrativa fragmentada e desfocada dos interesses e das necessidades da sociedade, evitando o desperdício de recursos públicos, reduzindo a superposição de ações e, consequentemente, aumentando a eficiência e a efetividade das políticas públicas existentes (Brasil, 2007).

Espera-se que o gestor de saúde local tenha um perfil articulador que possibilite o alcance de resultados positivos. De fato, a busca por inovação exige nova forma de se colocar diante do papel de ser gestor municipal de saúde (Fernandez e Mendes, 2007).

Espera-se que com gestores de saúde preparados, a operacionalização das políticas públicas flua no sentido de promover mudanças que impactem positivamente na qualidade de vida da população. Sabe-se que a Promoção da Saúde tem grande potencial para mobilizar a população no sentido de seu fortalecimento e impactar nos indicadores de saúde de forma positiva.

Lembrando que a PNPS determina como responsabilidades dos gestores municipais de saúde: a implementação das diretrizes de capacitação e educação permanente em consonância com as realidades locais; a viabilização de financiamento para a Promoção da Saúde dentro da política de educação permanente, bem como propõe instrumento de avaliação de desempenho, no âmbito municipal; o estabelecimento de mecanismos para a qualificação dos profissionais do sistema local de saúde para desenvolver as ações de Promoção da Saúde; a promo- 
ção da articulação intersetorial para a efetivação da Política Nacional de Promoção da Saúde; e a busca por parcerias governamentais e não governamentais para potencializar a implementação das ações de Promoção da Saúde no âmbito do SUS. Os gestores municipais de saúde precisam ter um papel mais articulador que facilite o desenvolvimento das ações de Promoção da Saúde no município (Brasil, 2007).

\section{Considerações Finais}

Conclui-se que os gestores de saúde dos municípios que compõem o CGR do Alto Capivari percebem as ações de Promoção da Saúde como ações de prevenção de doenças e estimulam ações de saúde voltadas para a mudança de comportamento e hábitos de vida.

A Promoção da Saúde, na perspectiva ampliada de saúde, como definida no âmbito do movimento da Reforma Sanitária Brasileira, do SUS e das Cartas de Promoção da Saúde, diz que os modos de viver não se referem apenas ao exercício da vontade e/ou liberdade individual e comunitária. Ao contrário, os modos como sujeitos e coletividades elegem determinadas opções de viver como desejáveis, organizam suas escolhas e criam novas possibilidades para satisfazer suas necessidades estão condicionados ao processo de construção e contexto da própria vida. Dessa maneira, operacionalizar ações de Promoção da Saúde na perspectiva dos gestores pressupõe que estes tenham um projeto para a construção e o fortalecimento do capital social em seu município, através da priorização de programas, projetos e ou atividades de impacto social.

As ações de Promoção da Saúde ainda são incipientes no SUS, que ainda se baseia em um modelo de saúde médico-centrado, especializado e com ações curativas. Os ACSs têm potencial para ajudar as comunidades e as pessoas a desenvolverem habilidades e o saber crítico de cuidar de sua saúde e lutar pelos seus direitos garantidos por lei.

Atualmente as iniciativas mais exitosas no campo da promoção da saúde têm sido circunscritas a iniciativas de Organizações Não Governamentais e a algumas prefeituras "iluminadas". Fomentar a capacitação e o envolvimento dos gestores públicos, para que estes possam investir na qualidade de vida dos cidadãos parece ser uma estratégia inteligente.

\section{Colaboração dos autores}

Fracolli participou da confecção inicial da pesquisa, coleta e análise dos dados, discussão e redação final do artigo. Gomes realizou a coleta dos dados, participou da análise dos dados e da discussão e redação final do artigo. Gryschek participou da discussão e redação final do artigo.

\section{Referências}

ALMEIDA, E. Z. As concepções do agente comunitário de saúde sobre promoção da saúde. 2008. Dissertação (Mestrado em Enfermagem) - Escola de Enfermagem da Universidade de São Paulo, São Paulo, 2008.

BARDIN, L. Análise de conteúdo. Lisboa: Edições 7O, 1970.

BRASIL. Ministério da Saúde; Ministério da Educação. Referencial curricular para o curso técnico de agente comunitário de saúde: área profissional. Brasília, DF, 2004.

BRASIL. Ministério da Saúde. Secretaria de Vigilância em Saúde. Secretária de Atenção à Saúde. Política nacional de promoção da saúde. Brasília, DF, 2007.

CHIESA, A. M.; FRACOLLI, L. A. O trabalho dos agentes comunitários de saúde nas grandes cidades: análise do seu potencial na perspectiva da promoção da saúde. Revista Brasileira Saúde da Família, Brasília, DF, v. 5, n. 7, p. 42-50, 2004.

CZERESNIA, D. O conceito de saúde e a diferença entre prevenção e promoção. In: CZERESNIA, D.; FREITAS, C. M. (Org.). Promoção da saúde: conceitos, reflexões, tendências. Rio de Janeiro: Fiocruz, 2003. p. 39-53.

FERNANDEZ, J. C. A.; MENDES, R. (Org.). Promoção da saúde e gestão local. São Paulo: Aderaldo \& Rothschild: Cepedoc, 2007.

GOMES, M. F. P. As potencialidades do agente comunitário de saúde para ações de promoção da saúde: em foco o Colegiado Gestor Regional de Alto Capivari. 2011. Dissertação (Mestrado em Enfermagem) - Escola de Enfermagem da Universidade de São Paulo, São Paulo, 2011. 
IBANEZ, N. et al. Política e gestão pública em saúde. Rio de Janeiro: Hucitec: CEALAG, 2011. MELO FILHO, D. A.; SÁ, R. F.; CHUMA, J. (Org.). Avaliação do capital social nas áreas de atuação do projeto municípios saudáveis no nordeste do Brasil. Recife: Bagaço, 2007.

PINTO, A. A. M. As potencialidades do agente comunitário de saúde na efetivação da promoção da saúde: uma análise das suas ações no município de Marília-SP. 2010. Dissertação (Mestrado em Enfermagem) - Escola de Enfermagem da Universidade de São Paulo, São Paulo, 2010.

SES - SECRETARIA DE ESTADO DA SAÚDE DE SÃO PAULO. Plano Estadual de Saúde 2012-2015. São Paulo, 2012.

SÁ, R. F.; NISHIDA, M. (Org.). Evidências de efetividade do projeto municípios saudáveis no nordeste do Brasil: o olhar da gestão. Recife: Editora Universitária da UFPE, 2008.
SANTOS, L. P. G. S. A atuação do agente comunitário de saúde em São Bernardo do Campo: possibilidades e limites para a promoção da saúde. 2005. Dissertação (Mestrado em Enfermagem) - Escola de Enfermagem da Universidade de São Paulo, São Paulo, 2005.

SÃO PAULO. Secretaria Estadual de Saúde. Regionais da saúde. São Paulo, 2012. Disponível em: <http://www.saude.sp.gov.br/ses/ institucional/departamentos-regionais-de-saude/ regionais-de-saude>. Acesso em: 10 mar. 2012.

SEADE - FUNDAÇÃO SISTEMA ESTADUAL DE ANÁLISE DE DADOS. Índice paulista de responsabilidade social: o estado dos municípios, 2008-2010. São Paulo, 2012. Disponível em: <http://www.iprsipvs.seade.gov.br/view/pdf/iprs/ IPRS_2012_V2_RMSP.pdf >. Acesso em: 12 jun. 2012. 